\title{
Hearts of Brazil Project
}

Raimundo Marques do Nascimento Neto, José Eduardo Krieger,

George L. Machado-Coelho, Alexandre da Costa Pereira

on behalf of the Investigators from the Hearts of Brazil Project

Knowledge of cardiovascular diseases has grown exponentially in the last decades due to an intensive multidisciplinary research program endeavored by the scientific community. Relevant aspects of the physiopathology of atherosclerosis and acute vascular occlusion have been provided by basic research. Epidemiological research, in turn, identified the importance of specific risk factors and greatly improved our tools to identify individuals with an increased risk of disease and/or cardiovascular events.

Finally, clinical trials have conclusively demonstrated which treatments work, and which ones do not ${ }^{1}$.

Nevertheless, one of the major existing criticisms is the little knowledge, by doctors and health care providers, of demographic data that are really representative of the current status of several pathologies in the Brazilian population. Despite the several tools that allow access to national data regarding the situation of several diseases in our population and among them, the cardiovascular ones, most national articles do not describe the country's reality or use statistics from other countries ${ }^{2}$.

In fact, several valuable consultation sources are available. Amongst them we could highlight the data from the national census made available by Instituto Brasileiro de Geografia e Estatística - the Brazilian Institute of Geography and Statistics - (IBGE) ${ }^{3}$, data from the Ministry of Health, through the information system of Sistema Unificado de Saúde - Unified Health System(Datasus) ${ }^{4}$ or data from Fundação Seade - Seade Foundation ${ }^{5}$. However, despite the relatively large amount of demographic and nosological data available for our population, specific data regarding the distribution and prevalence of diseases and cardiovascular risk factors for our population are relatively rare or are available only for specific groups or populations, in whom epidemiological studies on the prevalence of cardiovascular diseases have been performed.

Some examples of successful studies in our country are the ones carried out in Vitória, $\mathrm{ES}^{6}$, the Bambuí study, carried out in the town which bears its name ${ }^{7}$, a transversal study carried out amongst the population of the city of
Salvador, $\mathrm{BA}^{8}$, a transversal study carried out in Porto Alegre, $\mathrm{RS}^{9}$, among others. Nevertheless, data that can be utilized by other regions of the country or that disclose, at a national level, the real dimension of the existing interactions for cardiovascular risk amongst our population are not broadly available yet.

In this scenario, emerges the "Hearts of Brazil" project. The Hearts of Brazil project aims at establishing a profile of the cardiovascular risk distribution in the Brazilian population. With the data obtained through the Project, we expect that doctors and public health managers can choose more convenient and effective options for the Brazilian population in the several regions of the country.

A secondary objective of the project is to create a cardiovascular risk factors database that can be utilized openly by researchers interested in studying aspects that are still unknown regarding the epidemiology of cardiovascular risk factors in the Brazilian population.

\section{Study Design}

The project was developed through a pilot project conducted in the town of Ouro Preto, i.e., the Hearts of Ouro Preto Project. In the Hearts of Ouro Preto Project, the organization of questionnaires and field logistics were used and tested. We also learned how to obtain financial support for such projects. The Hearts of Brazil project is supported by several institutions, such as the Pharmaceutical Industry (Astra Zeneca, Biossintetica, Biolab, Libbs, Novartis and Sanofi), the equipment industry (Omron, Roche Diagnostic), the National Health Agency - ANS (Agência Nacional de Saúde), the Ministry of Health, the Conasems (Conselho Nacional de Secretários Municipais de Saúde - National Council of Municipal Secretaries of Health) and Furnas Electricity Center (Centrais Elétricas de Furnas).

All the logistic process and the results accomplished have attracted other possible partnerships for the project. All these resources and experience were conveyed to SBC-FUNCOR. The administrative structure and the capillarity of FUNCOR representation were crucial for the project success.

Mailing address: Raimundo Marques Nascimento Neto • Av. do Contorno, 3915 • 30320-450 • Belo Horizonte, MG - Brazil E-mail: rmnn@terra.com.br 
The study used a low-cost operational and financial methodology and was divided into two distinct phases.

At the first phase, through the use of structured questionnaires, socioeconomic, service and infrastructure information, as well as information on family characterization, nutritional status, use of medications, behavioral habits, history of cardiovascular risk factors and/or cardiovascular disease were obtained from approximately 2,500 individuals, randomly chosen in 72 Brazilian cities or towns.

The first phase sampling was carried out by the Vox Populi opinion-poll institute and its main objective was to guarantee that the sample obtained would be representative of the Brazilian population regarding its distribution by gender, age and socioeconomic status. Seventy-two cities or towns from the 5 regions of the country were chosen (Fig. 1) and the number of individuals sampled was proportional to the population residing in each city or town. This number ranged from 15 individuals for the smaller towns to 400 individuals for the city of São Paulo.

The project was approved by the National Commission of Ethics in Research - Comissão Nacional de Ética em Pesquisa (CONEP) and the first phase was initiated in
July 2004. This phase was carried out through home interviews conducted by professionals from the Vox Populi Institute. The choice of individuals was carried out in three different stages. At the appointed city or town, the censitary sectors to be sampled were chosen from data provided by the IBGE. In these sectors, a street was chosen for the beginning of the poll. A home was chosen to be sampled on this street, using simple randomization rules. Inside this home, the individual to be sampled was chosen based on predefined criteria and respecting the stratification by gender and age. If the chosen individual was not present at the moment of the interviewer's first contact, he/she could still be sampled at two subsequent visits carried out on different days and/or different times by the same interviewer. Strict sampling criteria guaranteed to the project that the sample studied was representative of the Brazilian population.

The second phase of the project consisted in inviting the individuals interviewed during the first phase to attend a medical examination in their respective cities or towns. During this phase, the project had the participation of several partners from SBC, as well as nurses, medical and nursing students, nurse aids, nurse technicians, and

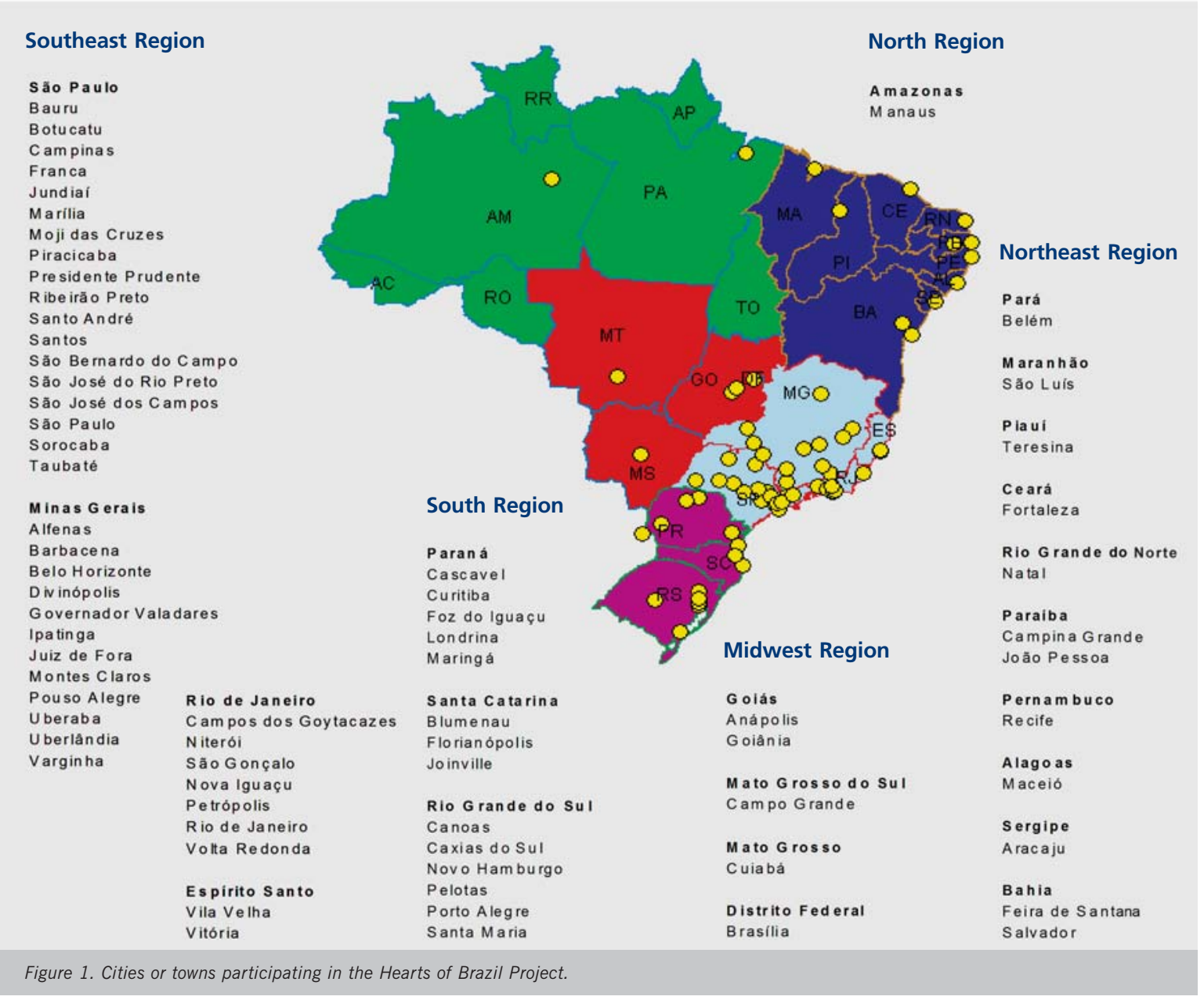


laboratory technicians, who were willing to welcome these individuals and carry out a standardized medical examination with the objective of acquiring data from medical questionnaires (non-applicable by nonmedical staff), clinical measurements such as arterial blood pressure, anthropometrical data, ankle-brachial index, and the collection of biological specimens for the evaluation of biochemical cardiovascular risks. Without the invaluable participation of the members of the Society who voluntarily devoted to carrying out the second phase, the Hearts of Brazil Project would have been but a recapitulation of data obtained from several other epidemiological studies in the Brazilian population.

In order to guarantee the uniformity of the data obtained in the 72 cities or towns where the project was carried out, standardized material for the collection of data such as arterial blood pressure measurements, anthropometrical data and biological material collection were carefully distributed to all participating centers. Also, training meetings were carried out with the presence of interviewers from all the centers, so that all the steps in this second phase of the project could be standardized.

At this step, data from 1,500 individuals were obtained representatively regarding gender, age and socioeconomic status. The difference regarding the number of individuals interviewed during the first phase in relation to the second does not constitute a significant bias and it was already expected, with the first phase being calculated taking into account the significant losses in the second phase.

This loss did not mean a loss of population representativeness regarding the variables gender and age range, as the organization of the project had a pro-active behavior by inviting individuals, during the second phase, from stratums that were underrepresented.

The participating individuals were evaluated in this second phase concerning variables of clinical measurements such as weight, height, abdominal circumference, etc. One of the most interesting data obtained at this second phase was the ankle-brachial index determination, an indicator of peripheral artery disease and a risk factor for coronary events yet to be studied regarding its distribution amongst the Brazilian population. Data originated from this phase will be used for the characterization of our population, as well as for the study of new interactions among the risk factors, probably specific ones, for the Brazilian population.

Finally, blood and urine samples were collected during this phase, for the determination of biochemical cardiovascular risk factors, such as fasting glycemia, total cholesterol and fractions, triglycerides, renal function data, and microalbuminuria. Through the Hearts of Brazil project, a DNA database has been established with the participating individuals' DNA samples, and it will be used to identify and characterize new risk factors that might explain in part the variability of cardiovascular disease in the Brazilian population.

\section{Using the data from the Hearts of Brazil Project}

Perhaps the major impact of the Project, as well as its biggest challenge, won't be attained through the direct information it provides on the distribution of cardiovascular risk factors, but through a better understanding of how these factors interact determining the risk of cardiovascular disease in our population.

This knowledge, however, will not be achieved through the simple description of the data obtained from the questionnaires and medical examination. It will come from a careful analysis based on scientific hypotheses that must be carried out using all the variables obtained from the Project. This is the major challenge of the Project from now on.

Thus, from a consensus among the several researchers who participated in the study design and data collection phases, as well as from debates with representatives from several departments of the Brazilian Society of Cardiology (SBC), it has been decided that all data obtained from the project will be made available to all those interested in developing research projects and who wish to utilize data from the Hearts of Brazil Project for their own projects. Similarly to other epidemiological studies, such as the MONICA Study from the World Health Organization or the American NHANES, researchers who are interested in the epidemiology of cardiovascular risk factors in Brazil will be able to request the release of such data by presenting a research project to the Management Committee of the Hearts of Brazil Project. The instructions are available at the Project site on the homepage of the Brazilian Society of Cardiology (http://www.cardiol.org). It is expected that the utilization of these data will be as broad as possible, and not restricted to a small number of investigators. We believe that only thorough a broad and stimulated use of the project data, its real contribution and comprehensiveness can be fully achieved.

\section{Conclusions}

It may seem, at first sight, that the data from the Hearts of Brazil project do not add any new important information on the problem of cardiovascular diseases in Brazil. We still need investments on basic research and clinical trials and particularly, we must have a large prospective cohort of cardiovascular disease conducted, which our country still lacks. Nevertheless, for its national features and comprehensiveness, the Hearts of Brazil Project has a crucial role on the definition of the model on which we must work this problem out. It is the responsibility of the Brazilian Society of Cardiology to see that such data are utilized democratically and comprehensively, and may add, in fact, invaluable information to the knowledge of cardiovascular disease determinants in our population. 


\section{REFERENCES}

1. Grover AS. Role of WHO-MONICA Project in unravelling of the cardiovascular puzzle. Lancet 2000;355:668-9.

2. Lotufo PA. Medicine based in brazilians. Arq Bras Cardiol 2001;76:419-20.

3. www.ibge.gov.br

4. www.datasus.gov.br

5. www.seade.gov.br

6. Bisi Molina M del C, Cunha R de S, Herkenhoff LF, Mill JG. Hypertension and salt intake in an urban population. Rev Saúde Pública 2003;37:743-50.
7. Barreto SM, Passos VM, Firmo JO, Guerra HL, Vidigal PG, Lima-Costa MF. Hypertension and clustering of cardiovascular risk factors in a community in Southeast Brazil - The Bambui Health and Ageing Study. Arq Bras Cardiol 2001;77:576-81.

8. Lessa I, Araújo MJ, Magalhães L, Almeida Filho N, Aquino E, Costa MC. Clustering of modifiable cardiovascular risk factors in adults living in Salvador (BA), Brazil. Rev Panam Salud Publica 2004;16:131-7.

9. Fuchs FD, Moreira LB, Moraes RS, Bredemeier M, Cardozo SC. Prevalence of systemic arterial hypertension and associated risk factors in the Porto Alegre metropolitan area. Population-based study. Arq Bras Cardiol 1994;63:473-9. 